\title{
Surface Excitations in a Bose-Einstein Condensate
}

\author{
R. Onofrio, D.S. Durfee, C. Raman, M. Köhl, C.E. Kuklewicz, and W. Ketterle \\ Department of Physics and Research Laboratory of Electronics, \\ Massachusetts Institute of Technology, Cambridge, MA 02139
}

(March 4, 2018)

\begin{abstract}
Surface modes in a Bose-Einstein condensate of sodium atoms have been studied. We observed excitations of standing and rotating quadrupolar and octopolar modes. The modes were excited with high spatial and temporal resolution using the optical dipole force of a rapidly scanning laser beam. This novel technique is very flexible and should be useful for the study of rotating Bose-Einstein condensates and vortices.

03.75.Fi, 67.40.Db, 67.57.Jj, 32.80.Lg
\end{abstract}

Elementary excitations play a crucial role in the understanding of many-body quantum systems. Landau derived the properties of superfluid liquid helium from the spectrum of collective excitations [i]. After the observation of Bose-Einstein condensation in dilute alkali gases [2], considerable theoretical and experimental efforts focused on collective excitations. This has already led to advances in our understanding of the weakly interacting Bose gas [3]. In most studies, collective modes were excited by modulating the parameters of the magnetic trapping potential [4.5. This method of exciting collective modes is limited to spatial perturbations that reflect the geometry of the trapping coils. Such a limitation is particularly severe for the widely used dc magnetic traps, where only modes with cylindrical symmetry have been excited [6].

Studies of high multipolarity modes are important for a number of reasons. First, high multipolarity modes are the closest counterpart to the surface excitations in mesoscopic liquid helium droplets. These surface modes are considered crucial to understand finite size effects in superfluids, but are difficult to achieve experimentally [7]. Second, for higher angular momentum the surface modes change their character from collective to single particle type [3]. This crossover could be crucial for the existence of a critical rotational velocity for vortex formation [8,9]. Also, because the thermal atoms are localized around the Thomas-Fermi radius, surface modes should be more sensitive to finite temperature effects [8].

In this Letter we report on the observation of surface excitations of a Bose-Einstein condensate confined in a dc magnetic trap. The excitations were induced by the optical dipole force of a focused red-detuned laser beam which was controlled by a 2-axis acousto-optic deflector. With these tools, local and controllable deformations of the magnetic trapping potential with both arbitrary spatial symmetry and timing can be achieved. This opens the way to selectively excite modes with higher multipolarity and complex spatial patterns.

Elementary excitations in a dilute Bose condensate are usually described by the hydrodynamic equations derived from the Bogoliubov theory [10], which closely resemble the equations describing superfluids at zero temperature [11]:

$$
m \frac{\partial}{\partial t} \mathbf{v}+\nabla\left(\frac{1}{2} m v^{2}+V_{e x t}(\mathbf{r})-\mu+\frac{4 \pi \hbar^{2} a}{m} \rho\right)=0 .
$$

Here $\rho(\mathbf{r}, t)$ and $\mathbf{v}(\mathbf{r}, t)$ are the condensate density and velocity respectively (linked by a continuity equation), $m$ the atomic mass, $a$ the $s$-wave scattering length, $\mu$ the chemical potential, and $V_{e x t}$ the external trapping potential. For an isotropic harmonic oscillator potential $V_{\text {ext }}=m \omega_{0}^{2} r^{2} / 2$ the solution for the density perturbation $\delta \rho$ can be expressed as:

$$
\delta \rho(\mathbf{r})=P_{\ell}^{(2 n)}(r / R) r^{\ell} Y_{\ell m}(\theta, \phi),
$$

where $P_{\ell}^{(2 n)}(r / R)$ are polynomials of degree $2 n$ ( $R$ being the Thomas-Fermi radius $R=\sqrt{2 \mu / m \omega_{0}^{2}}, Y_{\ell m}(\theta, \phi)$ are the spherical harmonics, and $\ell, m$ are the total angular momentum of the excitation and its $z$ component, respectively. The dispersion law for the frequency of the normal modes is expressed in terms of the trapping frequency $\nu_{0}=\omega_{0} / 2 \pi$ as 12]:

$$
\nu(n, \ell)=\left(2 n^{2}+2 n \ell+3 n+\ell\right)^{1 / 2} \nu_{0},
$$

which should be compared to the prediction for an ideal Bose gas in a harmonic trap, $\nu_{H O}=(2 n+\ell) \nu_{0}$. The effect of interactions in determining the transition from a collective to a single-particle regime is particularly evident for the excitations whose radial dependence of the density perturbation has no nodes $(n=0)$. These modes are referred to as surface excitations since the density perturbation, while vanishing at the origin, is peaked at the surface of the condensate. In thin films of superfluid liquid ${ }^{4} \mathrm{He}$ and ${ }^{3} \mathrm{He}$, their study has led to the observation of third sound 13. In a semiclassical picture these excitations can be considered the mesoscopic counterpart to tidal waves at the macroscopic level [14,15].

The experimental results were obtained using a newly developed apparatus for studying Bose-Einstein condensates of sodium atoms. A Zeeman slower with magnetic 
field reversal [16] delivers $10^{11}$ slow atoms s ${ }^{-1}$ which are collected in a magneto-optical (MOT) trap. A loading time of $3 \mathrm{~s}$ allowed us to obtain $10^{10}-10^{11}$ atoms in a dark-SPOT trap 117 at $\simeq 1 \mathrm{mK}$. After $5 \mathrm{~ms}$ of polarization gradient cooling, atoms in the $F=1, m_{F}=-1$ ground state at $\simeq 50-100 \mu \mathrm{K}$ are loaded into a magnetic trap. The latter realizes a Ioffe-Pritchard configuration modified with four Ioffe bars and two strongly elongated pinch coils, symmetrically located around a quartz glass cell. This novel design combines excellent optical access with tight confinement. The typical values for the axial curvature and radial gradient of the magnetic field at the trap center are $202 \mathrm{G} / \mathrm{cm}^{2}$ and $330 \mathrm{G} / \mathrm{cm}$, among the largest ever obtained in such magnetic traps. The resulting trapping frequencies are $\nu_{r}=547 \mathrm{~Hz}$ and $\nu_{z}=26$ $\mathrm{Hz}$ for the radial and the axial directions respectively. The background gas-limited lifetime of the atoms in the magnetic trap at $\simeq 10^{-11}$ Torr is around $1 \mathrm{~min}$. After evaporative cooling with an rf-sweep lasting $20 \mathrm{~s}$, around $5-10$ million atoms are left in a condensate with a chemical potential of $200 \mathrm{nK}$ and a negligible thermal component (condensate fraction $\geq 90 \%$ ). A decompression to the final radial and axial trapping frequencies of $(90.1 \pm 0.5) \mathrm{Hz}$ and $18 \mathrm{~Hz}$ lowered the density to $2 \cdot 10^{14}$ $\mathrm{cm}^{-3}$ where three-body recombination losses were less prominent. The radial trapping frequency was measured by exciting the condensate motion with a short modulation of the bias magnetic field and looking at the free center of mass oscillation in the magnetic trap.

Surface modes were excited by perturbing the magnetic trapping potential with light from a Nd:YAG laser (emitting at $1064 \mathrm{~nm}$ ) travelling parallel to the axis of the trap and focused near the center of the magnetic trap. Because of the low intensity of the laser beam and the large detuning from the sodium resonance, heating from spontaneous scattering was negligible. The laser beam was red-detuned from the sodium resonance and therefore gave rise to an attractive dipole potential 18. The $1 \mathrm{~mm}$ Rayleigh range of the beam waist is considerably longer than the $220 \mu \mathrm{m}$ axial extent of the condensate. Therefore, the laser only created radial inhomogeneities in the trapping potential, leaving the axial motion almost undisturbed.

The spatial and temporal control of the beam was achieved with two crossed acousto-optic deflectors. Using the 2-axis deflector arbitrary laser patterns could be scanned in a plane transverse to the propagation of the laser beam. The maximum size of these patterns is 100 beam widths in both directions. The scan rate was chosen to be $10 \mathrm{kHz}$, which is much larger than the trapping frequencies. Thus, the atoms experienced a timeaveraged potential that is superimposed upon the magnetic trap potential as depicted in Fig. 1a. For these experiments a beam width of $15 \mu \mathrm{m}$ and a power of $80 \mu \mathrm{W}$ were used to generate a potential depth corresponding to $20 \%$ of the chemical potential for each point.

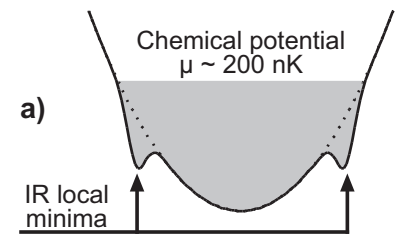

b)

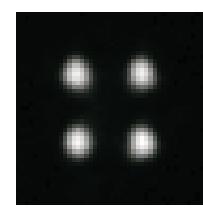

FIG. 1. Scheme for exciting collective modes by optically deforming the magnetic trap potential. (a) The combined potential of the magnetic trap and two red-detuned beams. (b) An image of the four-point IR pattern taken with a CCD camera. The laser beams were arranged on the corners of a $40 \mu \mathrm{m} \times 40 \mu \mathrm{m}$ square centered on a condensate with a Thomas-Fermi radius of $\simeq 25 \mu \mathrm{m}$.

For an anisotropic axially symmetric trapping potential, only the $z$ component of the angular momentum is conserved and the eigenfunctions are more complicated than in the isotropic case. However, surface modes of the form as in Eq. (2) with $m= \pm \ell$ are still solutions with a frequency [12]:

$$
\nu(m= \pm \ell)=\sqrt{\ell} \nu_{r}
$$

Quadrupolar standing waves were studied by exciting a superposition of $\ell=2, m=2$ and $\ell=2, m=-2$ modes with a pattern of two points located on opposite sides of the condensate. The light intensity was modulated in phase at the expected quadrupole frequency $\nu_{2}=\sqrt{2} \nu_{r}$. After 5 cycles the IR light was turned off, leaving the condensate free to oscillate in the magnetic trap. The condensate was then released from the magnetic trap and after $20 \mathrm{~ms}$ of ballistic expansion it was probed by resonant absorption imaging along the axis of the trap. In Fig. 2a, images are shown for different phases of the oscillation. The aspect ratio of the condensate oscillates at a frequency of $(130.5 \pm 2.5) \mathrm{Hz}$ with a damping time of about $0.5 \mathrm{~s}$. A similar damping time was observed for the lowest $m=0$ mode of an almost pure condensate [19].

A rotating wave $\ell=2, m=2$ was excited with two IR spots of constant intensity rotating around the axis at half the measured quadrupole frequency. This excitation scheme was highly frequency selective. When the rotation frequency deviated by $10 \%$ from the resonance no excitation of this mode was observed, consistent with the narrow bandwidth of the mode. In Fig. 2b we show a set of 10 pictures of the rotating mode taken with nondestructive phase-contrast imaging [20] in the magnetic trap.

The higher lying $\ell=4$ surface mode (superposition of $m= \pm 4$ ) was driven with a four-point pattern that was intensity-modulated at the expected frequency $\nu_{4}=2 \nu_{r}$ (Fig. 1b). In Fig. 3a time of flight absorption images are shown for variable hold times in the magnetic trap after stopping the drive and compared to the time evolution for a pure $\ell=4$ surface mode (Fig. 3b). By analyzing the density distribution close to the surface we extracted 
the Fourier spectrum of the first radial moment $r=r(\theta)$, which was strongly peaked at $\ell=4$. The time evolution of the $\ell=4$ Fourier cosine coefficient was obtained by repeating the analysis for various hold times (Fig. 3c).

a)

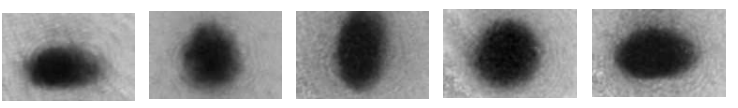

b)

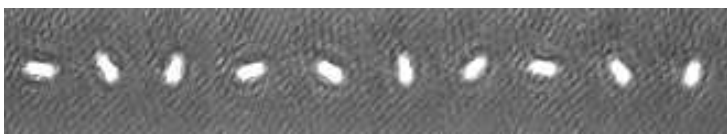

FIG. 2. Observation of standing and rotating surface waves. (a) shows absorption images of a standing $\ell=2, m= \pm 2$ quadrupole mode. They were taken after free oscillation times of $3.5,5.25,7,8.75$ and $10.5 \mathrm{~ms}$ (from left to right) in the magnetic trap and $20 \mathrm{~ms}$ of ballistic expansion. In (b) multiple phase-contrast images of a clockwise rotating $\ell=2, m=2$ quadrupole surface excitation are shown, each frame being $2 \mathrm{~ms}$ apart. The field of view of each image is $720 \mu \mathrm{m} \times 480 \mu \mathrm{m}$.

a)

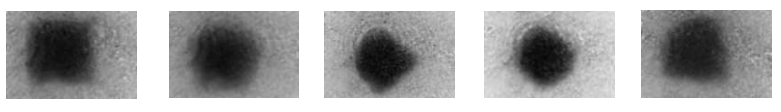

b)

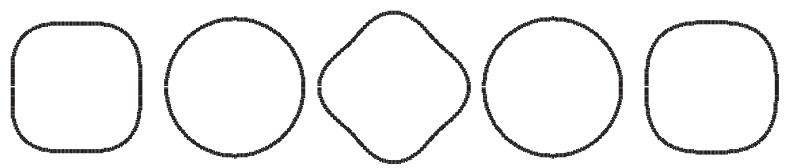

c)

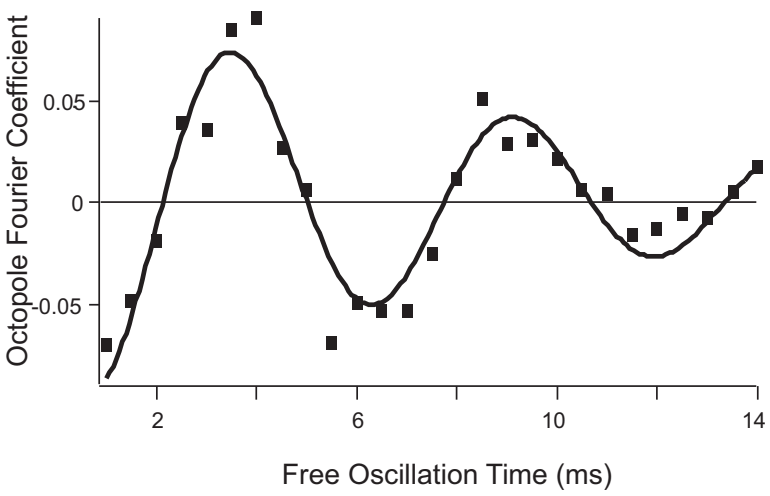

FIG. 3. Observation of an octopolar mode of a Bose-Einstein condensate. Absorption images of a condensate for various hold times $(1,2,3.5,4.5$ and $6.5 \mathrm{~ms}$ from left to right) in the magnetic trap (a) after the excitation. The shape of a pure $\ell=4$ oscillation is schematically depicted in (b) for one cycle. (c) shows the $\ell=4$ cosine Fourier coefficient as a function of the free oscillation time in the magnetic trap. The field of view of each image is $720 \mu \mathrm{m} \times 480 \mu \mathrm{m}$.

We observed an exponentially decaying oscillation at $\nu_{4}=(177 \pm 5) \mathrm{Hz}$ with a damping time of $\tau_{4}=(9.5 \pm$ 2.2) ms. The agreement between the measured fre- quencies and the hydrodynamic predictions is very good (see Table I). Note that the octopolar mode is damped much faster than the quadrupole mode. This could indicate that higher order surface excitations interact more strongly with the thermal cloud. Due to the mean-field repulsion of the condensate, the effective potential felt by the thermal atoms has a minimum at the ThomasFermi radius. For increasing $\ell$ surface waves are more localized in the same region (Eq.(2)). Thus, a systematic study of the temperature dependence of frequencies and damping times of higher order surface modes could extend thermometry for Bose-Einstein condensates to lower temperatures where no thermal cloud is discernible (and the usual method of fitting the wings of the thermal distribution is no longer applicable).

\begin{tabular}{|c|r|c|c|}
\hline$\ell$ & $\nu_{\ell}(\mathrm{Hz})$ & $\nu_{\ell} / \nu_{1}(\exp )$ & $\nu_{\ell} / \nu_{1}(t h)$ \\
\hline 1 & $90.1 \pm 0.5$ & - & - \\
2 & $130.5 \pm 2.5$ & $1.45 \pm 0.04$ & $\sqrt{2}$ \\
4 & $177 \pm 5$ & $1.96 \pm 0.06$ & 2 \\
\hline
\end{tabular}

Table I: Comparison between observed and predicted frequencies for the quadrupole and the octopole surface excitations, normalized to the radial trapping frequency (dipole mode) $\nu_{1}$.

For higher $\ell$, the crossover from the hydrodynamic regime to the single particle picture could be explored. This is expected to occur for $\ell \geq$ $\ell_{\text {crit }}=2^{1 / 3}\left(R / a_{H O}\right)^{4 / 3} \simeq 24$ [8] (where $a_{H O}=$ $\left[\hbar / 2 \pi m\left(\nu_{r}^{2} \nu_{z}\right)^{1 / 3}\right]^{1 / 2}$ is the harmonic oscillator length), for our trap parameters. The excitation of such modes would require smaller beam waists. However, this resulted either in a very weak excitation or, when the power was increased, the condensate became strongly distorted and/or localized around the laser focus leading to high densities and to large recombination losses. We plan to use blue-detuned light in the future, which will make it easier to create stronger perturbations without loss mechanisms.

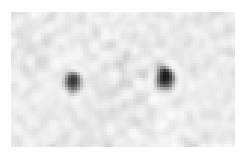

a)

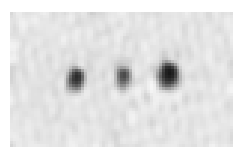

b)

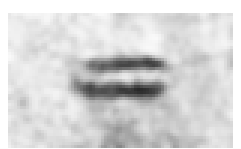

c)
FIG. 4. Bose-Einstein condensates in time-averaged potential optical traps. Patterns with two (a) and three (b) points and a double sheet (c) are shown. The absorption images are taken along the axis of the laser beam, with resonant probe light. The frame size is $200 \mu \mathrm{m} \times 120 \mu \mathrm{m}$.

Our method of generating time-averaged optical potentials can also be used to create purely optical traps in a variety of geometries. By increasing the laser intensity 
and shutting off the magnetic trap we were able to tranfer the condensate into multiple optical dipole traps, as shown in Fig. 4. They can be used for interference of multiple condensates and studies of coherence and decoherence.

Another interesting possibility is the study of condensates in rotating potentials where vortices should be stable. Our first attempts showed a very short trapping time, probably caused by heating due to micromotion. It should be possible to overcome this limitation by increasing the scan frequency beyond the current maximum value of $100 \mathrm{kHz}$.

In conclusion, we have developed a technique to excite surface modes in a Bose-Einstein condensate by inducing deformations of the trap potential with a rapidly scanning red-detuned laser beam. With this technique we could excite both standing and rotating modes. The measured frequencies for quadrupole and octopole modes are in agreement with the predictions of the hydrodynamic theory for collective excitations of dilute Bose gases. This flexible technique should be useful for the investigation of the interplay between collective excitations and the physics of rotating Bose-Einstein condensates.

We would like to thank J. Gore, Z. Hadzibabic, and J. Vogels for experimental assistance and useful discussions. This work was supported by the ONR, NSF, JSEP (ARO), NASA, and the David and Lucile Packard Foundation. M.K. acknowledges also support from Studienstiftung des Deutschen Volkes.

[1] L. D. Landau, J. Phys. USSR 5, 71 (1941).

[2] M. H. Anderson et al., Science 269, 198 (1995); K. B. Davis et al., Phys. Rev. Lett. 75, 3969 (1995); C. C. Bradley et al., Phys. Rev. Lett. 78, 985 (1997).

[3] F. Dalfovo, S. Giorgini, L. P. Pitaevskii, S. Stringari, Rev. Mod. Phys. 71, 463 (1999).

[4] D. S. Jin, J. R. Ensher, M. R. Matthews, C. E. Wieman, and E. A. Cornell, Phys. Rev. Lett. 77, 420 (1996).

[5] M.-O. Mewes, M. R. Andrews, N. J. van Druten, D. M. Kurn, D. S. Durfee, C. G. Townsend, and W. Ketterle, Phys. Rev. Lett. 77, 988 (1996).

[6] The time-dependent potential of the TOP-trap allowed the excitation of $m=2$ modes 4 .

[7] S. A. Chin and E. Krotscheck, Phys. Rev. Lett. 74, 1143 (1995); D.L. Whitaker, C. Kim, C.L. Vincente, M. A. Weilert, H.J. Maris, and G.M. Seidel, J. Low Temp. Phys. 113, 491 (1998).

[8] F. Dalfovo, S. Giorgini, M. Guilleumas, L. P. Pitaevskii, and S. Stringari, Phys. Rev. A 56, 3840 (1997).

[9] E. C. Lundh, C. J. Pethick, and H. Smith, Phys. Rev. A 55, 2126 (1997).

[10] N. N. Bogoliubov, J. Phys. USSR 11, 23 (1947).

[11] P. Nozières and D. Pines, The Theory of Quantum Liq- uids (Addison-Wesley, Reading, MA, 1990), Vol. II.

[12] S. Stringari, Phys. Rev. Lett. 77, 2360 (1996).

[13] C.W.F. Everitt, K.R. Atkins, and A. Denenstein, Phys. Rev. 136, A1494 (1964); A.M.R. Schechter, R.W. Simmonds, R.E. Packard, and J.C. Davis, Nature 396, 555 (1998).

[14] I. Newton, Mathematical Principles of Natural Philosophy [English translation by F. Cajori, University of California Press, Berkeley, 1947], p. 435.

[15] U. Al Khawaja, C.J. Pethick, and H. Smith, Phys. Rev. A 60, 1507 (1999).

[16] A. Witte, Th. Kisters, F. Riehle, and J. Helmcke, J. Opt. Soc. Am. B 9, 1030 (1992).

[17] W. Ketterle, K. B. Davis, M. A. Joffe, A. Martin, and D. E. Pritchard, Phys. Rev. Lett. 70, 2253 (1993).

[18] S. Chu, J.E. Bjorkholm, A. Ashkin, and A. Cable, Phys. Rev. Lett. 57, 314 (1986).

[19] D. M. Stamper-Kurn, H.-J. Miesner, S. Inouye, M. R. Andrews, and W. Ketterle, Phys. Rev. Lett. 81, 500 (1998).

[20] M. Andrews, M.-O. Mewes, N. J. van Druten, D. S. Durfee, D. M. Kurn, and W. Ketterle, Science 273, 84 (1996). 